\title{
Quantification of thermal spread and burst pressure after endoscopic vessel harvesting: A comparison of 2 commercially available devices
}

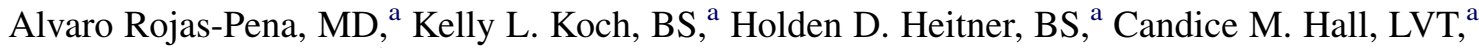 \\ Ingrid L. Bergin, VMD, ${ }^{b}$ and Keith E. Cook, $\mathrm{PhD}^{\mathrm{a}}$
}

\begin{abstract}
Objective: Endoscopic vein harvesting systems have grown in popularity and are becoming the gold standard for coronary artery bypass grafting. Although a consensus is present that endoscopic vessel harvesting minimizes wound complications, long-term graft patency remains a concern. It has been proposed that endoscopic vessel harvesting affects graft patency because of irreversible trauma to the endothelium. This study was performed to examine the extent of thermal injury caused by 2 commercially available endoscopic vessel harvesting systems in a porcine model.
\end{abstract}

\begin{abstract}
Methods: Superficial epigastric veins and saphenous arteries were exposed in 10 anesthetized swine. All vessel samples (conduits) were harvested randomly with either a VirtuoSaph (Terumo Cardiovascular, Ann Arbor, Mich) or VASOVIEW 6 (MAQUET, Inc, Wayne, NJ) endoscopic vessel harvesting system. Conduits were harvested and saved for either histologic analysis or burst-pressure test. Statistical differences were analyzed by using a Wilcoxon rank sum test in SAS 9.2 software (SAS Institute, Inc, Cary, NC) for thermal spread and a 2-tailed $t$ test with equal variance for burst pressure.
\end{abstract}

Results: The average thermal spreads for saphenous artery and superficial epigastric vein conduits were significantly shorter in the VirtuoSaph group $(0.42 \pm 0.08$ and $0.49 \pm 0.05 \mathrm{~mm}$, respectively) than in the VASOVIEW 6 group $(1.05 \pm .04$ and $0.94 \pm 0.19 \mathrm{~mm}$, respectively). No significant differences were observed in burst pressure.

Conclusions: The length of thermal spread is short in arterial and venous conduits $(0.4-1.1 \mathrm{~mm})$ and depends on the endoscopic vessel harvesting system. Clinical protocols should include a minimal length of the cauterized branch to ensure that thermal spread does not reach the main vessel. The results of this study suggest that at least $1 \mathrm{~mm}$ is sufficient. (J Thorac Cardiovasc Surg 2011;142:203-8)

For the last 20 years, endoscopic vessel harvesting (EVH) has grown in popularity and is becoming the method of choice for coronary artery bypass grafting (CABG) and lower limb revascularizations. ${ }^{1}$ The use of EVH had been reported to reduce postoperative leg wound complications, including infection, and improved patient satisfaction compared with the conventional (open) harvesting technique. ${ }^{2}$ In 2005, the International Society for Minimally Invasive Cardiothoracic Surgery published a consensus statement recommending that endoscopic harvesting be the standard of care. ${ }^{3}$ The reason for this consensus is that $\mathrm{EVH}$

\footnotetext{
From the General Surgery Department, ${ }^{\mathrm{a}}$ ECMO Laboratory, University of Michigan Health System, and the Lab Animal Medicine Unit, ${ }^{\mathrm{b}}$ University of Michigan Medical School, Ann Arbor, Mich.

Disclosures: Materials and endoscopic vessel harvesting products were provided by Terumo Cardiovascular Systems Corporation (Ann Arbor, Mich) and support was provided by the Michigan Institute for Clinical and Health Research (MICHR), grant UL1RR024986, for their biostatistical advice. Dr Rojas-Pena is a consultant for Terumo Cardiovascular Systems Corporation.

Received for publication June 2, 2010; revisions received Aug 26, 2010; accepted for publication Sept 17, 2010; available ahead of print Dec 6, 2010.

Address for reprints: Alvaro Rojas-Pena, MD, University of Michigan, $1150 \mathrm{~W}$ Medical Center Dr, MSRB II-Room B570E, Ann Arbor, MI 48109 (E-mail: alvaror@ umich.edu).

0022-5223/\$36.00

Copyright (C) 2011 by The American Association for Thoracic Surgery

doi:10.1016/j.jtcvs.2010.09.055
}

minimizes leg complications, wound complications, or both. Complication rates reported in this study were $4 \%$ with EVH versus $19 \%$ with the open (longitudinal) technique. ${ }^{4}$ On the other hand, long-term graft patency and irreversible injury to the vascular endothelium of the grafts are concerns with EVH. ${ }^{5,6}$ In 2009, Lopes and colleagues ${ }^{7}$ reported a $47.7 \%$ rate of vein graft failure at 12 to 18 months when the endoscopic technique was used versus $38 \%$ with the open technique. Furthermore, these findings correlated with adverse clinical outcomes (death, myocardial infarction, or repeat revascularization) at 3 years. Other studies, however, saw vein graft occlusion rates at 6 months between $22 \%$ for EVH and $18 \%$ for the open technique, and 1-year patency rates of $89 \%$ for both methods without significant differences between techniques. ${ }^{3,8,9}$

It has been proposed that $\mathrm{EVH}$ causes a reduction in graft patency because of direct and irreversible trauma to the endothelium at the time of harvesting. ${ }^{10-12}$ Disruption and damage of the vascular endothelium might lead to exposure of smooth muscle and connective tissue that then leads to local coagulation. Brown and colleagues ${ }^{13}$ have shown that residual clot strands within conduits excised by using the EVH technique play an important role in graft patency, and thus systemic heparinization has been suggested as a benign change in EVH practice. One possible cause of 


\section{Abbreviations and Acronyms \\ $\mathrm{CABG}=$ coronary artery bypass grafting \\ $\mathrm{EVH}=$ endoscopic vessel harvesting \\ SA $=$ saphenous artery \\ $\mathrm{SEV}=$ superficial epigastric vein}

endothelial damage is thermal injury to the grafts during branch ligation. This damage has been quantified or measured objectively by very few studies, and the majority of them use subjective numeric grading scales/damage scores. ${ }^{9,14-16}$ The strength of the vascular seal, as well as the extent of thermal damage, has not been well quantified. Thus the impetus for this study was the advent of newer technology that aims to decrease thermal spread while maintaining a reliable and repeatable vascular seal.

Therefore further investigation is required to determine the degree of thermal damage during EVH. This study was performed to compare the extent of thermal injury caused by 2 commercially available EVH devices during artery and vein harvesting in a porcine model. In addition, the quality of the seal was assessed to determine whether a more thorough seal might lead to a greater degree of thermal damage.

\section{MATERIALS AND METHODS \\ EVH}

All pigs received humane care in compliance with the "Guide for the care and use of laboratory animals." The protocol was approved by the University Committee on Use and Care of Animals at the University of Michigan. Ten healthy pigs (approximately $35 \mathrm{~kg}$ ) were sedated with an intramuscular mix of $5 \mathrm{mg} / \mathrm{kg}$ tiletamine $\mathrm{HCl}$ and zolazepam $\mathrm{HCl}$ (Telazol; Wyeth Holdings Corp, Carolina, Puerto Rico) and $3 \mathrm{mg} / \mathrm{kg}$ xylazine (Tranquived; Vedco, Inc, St Joseph, Mo). A 7.0 to 8.0 ID cuffed endotracheal tube (Mallinckrodt, St Louis, Mo) was used for intubation, and the swine were ventilated with $1 \%$ to $3 \%$ isoflurane (Hospira, Inc, Lake Forest, Ill) in $100 \%$ oxygen. Mechanical ventilation settings were adjusted to maintain normal end-tidal $\mathrm{CO}_{2}$ and arterial blood gas levels.

After skin cut-down at the right side of the neck, the common carotid artery and internal jugular vein were used to advance catheters for hemodynamic monitoring and fluid administration, respectively. The arterial and venous pressure lines were connected to fluid pressure transducers (Abbot Critical Care Systems, North Chicago, Ill) and monitored continuously on a Series 7000 Pressure Monitor (Marquette Electronics, Milwaukee, Wis). The left and right superficial epigastric veins (SEVs) and left and right saphenous arteries (SAs) were surgically exposed about 20 to $25 \mathrm{~cm}$ by using an open technique, and each vessel was covered with moist gauze until further harvesting and division. The diameter of the SAs was between 1.10 and $1.25 \mathrm{~mm}$, and that of the SEVs was between 0.25 and $0.63 \mathrm{~mm}$. These vessels were chosen to mimic the branches of the main human saphenous vein and radial artery because their sizes are similar to these branches.

All vessel samples (conduits) were harvested randomly with either a VirtuoSaph (Terumo Cardiovascular, Ann Arbor, Mich) or VASOVIEW 6 (MAQUET, Inc, Wayne, NJ) EVH system. The VASOVIEW 6 Bisector uses 2 equally sized electrodes for sealing the branch and a mechanical blade for cutting it. The VirtuoSaph System device has 2 electrodes (1 small and 1 large) but no mechanical cutting element because the branch is sealed and cut solely through the application of electrosurgical energy. Each de- vice is used with an electrosurgical generator, but the VASOVIEW 6 device is operated by using the coag mode, and the VirtuoSaph device is operated by using the cut mode.

Mechanical variables, such as stretch and physical trauma, were minimized during the dissection by using an open dissection technique. Thus the $2 \mathrm{EVH}$ devices were used only to divide the conduit side branches and cut multiple conduits from each vessel. Conduits (12-15 per vessel) of 10 to $12 \mathrm{~mm}$ in length were harvested (mimicking branches of the human saphenous vein and radial artery). During harvesting, 3 seconds of energy were applied for each sample, which were then saved for either histologic analysis or burst pressure. Before all cases, the operator/harvester (A.R.P.) was trained and certified in the use of both devices. Dr Rojas-Pena has experience in using EVH devices in animal settings for training and research only. All cases used the same operator. Eight pigs were used to harvest vessel samples for histomorphometric evaluation, and an additional pig in each group was used to determine seal quality based on burst pressure. The following numbers of conduits were harvested per vessel type and EVH device for histologic analysis $(n=4$ pigs per group): VirtuoSaph, 144 conduits (61-SA and 83-SEV); VASOVIEW 6, 132 conduits (53-SA and 79-SEV).

\section{Tissue Analysis}

For histomorphometric evaluation, conduits were immediately fixed in a numbered vial with $10 \%$ formalin. They were then embedded longitudinally in paraffin, cut, and stained with a standard hematoxylin and eosin stain. Samples were then analyzed by a blinded veterinary pathologist (I.L.B.) with a BX45 Universal Infinity System (Olympus Corp, Tokyo, Japan) light microscope with an attached DP72 12.8 megapixel digital camera (Olympus Corp) and DP2-TWAIN software (Olympus Corp, Version 02.01). Digital photomicrographs were taken of cautery damage at $40 \times$ and $100 \times$ total magnification, and measurements were made from these photomicrographs (Figure 1). Histologic changes attributed to cautery consisted of coagulative necrosis affecting the tunica adventitia, media, and/or intima. All visible areas of coagulative necrosis within the vascular wall or in immediate contact (intimal) with the vascular wall were included, and the maximal longitudinal extent of damage was recorded. Maximal length of thermal spread was defined as the length from the cut edge to the point/boundary where tissue was undamaged. Where the endothelium could be clearly visualized, the extent of endothelial damage correlated approximately with the extent of adventitial and medial damage (Figure $1, A$ ). In addition, damage was classified as primarily adventitial (Figure 1,B) or affecting the tunica adventitia, media, and intima (Figure 1,C). Coagulation necrosis was most consistently evident in the adventitial and medial layers of the vascular wall.

For burst-pressure studies, conduits were individually placed in $0.9 \%$ normal saline vials immediately after harvesting and tested the following morning. Burst pressure tests were performed by a blinded laboratory technician. Conduits were removed from the vials with forceps while only touching the cut end of the vessel. The cut end was then cannulated with a 15-gauge dispensing tip (Nordson EFD, East Providence, RI), and a softjaw mosquito clamp was used to clamp the conduit to the dispensing tip. A 60-mL syringe was filled with $0.9 \%$ saline solution, attached to the dispensing tip, and inserted into a multiple-syringe pump (MSP-DT2; AS ONE, Osaka, Japan; Figure 2). The syringe pump injected fluid at a rate of 360 $\mathrm{mL} / \mathrm{h}$ while a pressure gauge PG-200/PG-208 (Copal Electronics, Tokyo, Japan) recorded the vessel pressure with Wavelogger software (NR-TH08; Keyence, Osaka, Japan). Both data acquisition and the syringe pump were stopped once vessel leak/burst was observed. Burst pressure was defined as the maximum pressure needed to break a vessel's thermal seal.

\section{Statistical Analysis}

The extent of the thermal spread and burst pressure were analyzed as outcome measures. Thermal spread results were not normally distributed. Therefore thermal-spread results were separated into categories of either 


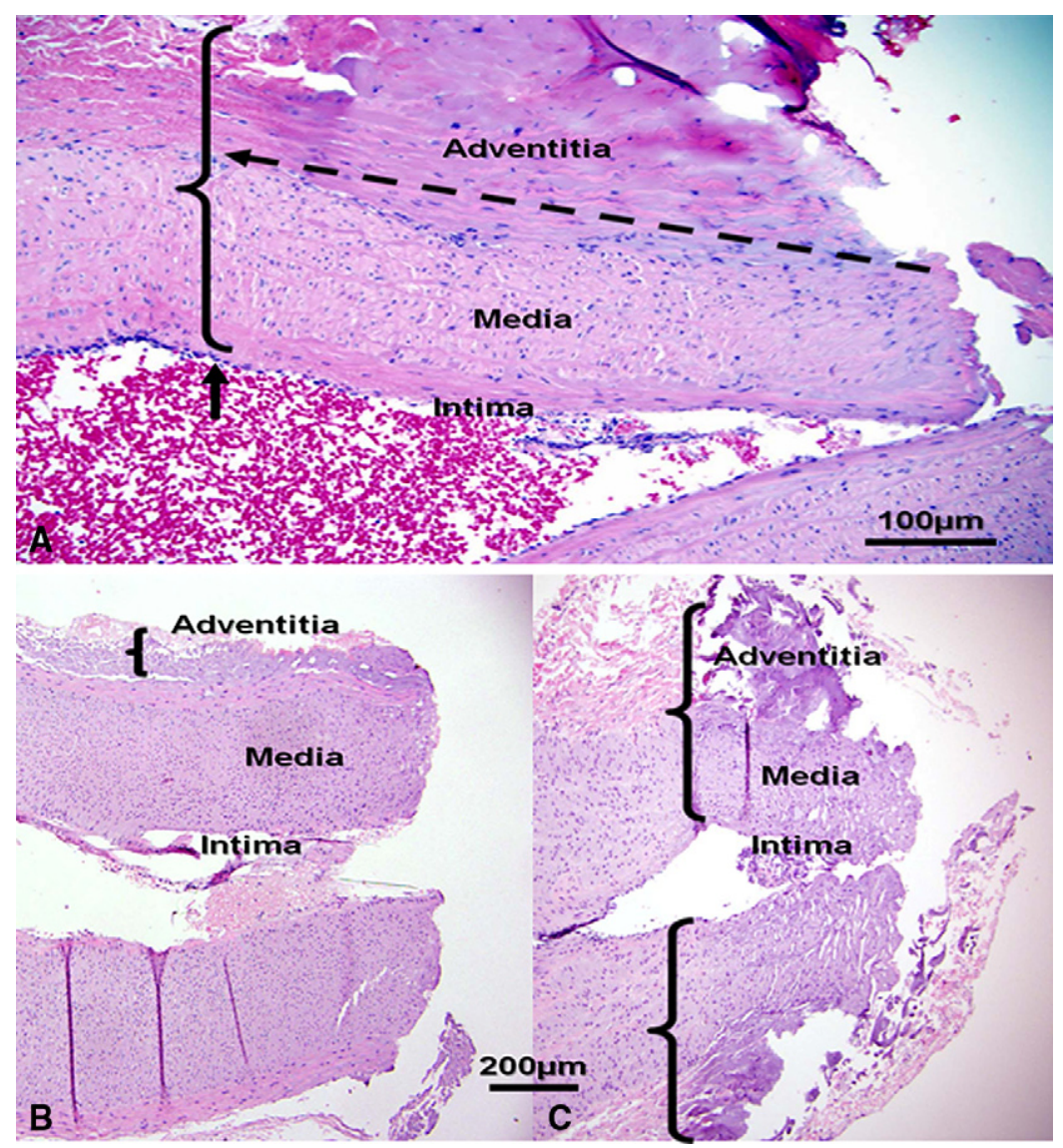

FIGURE 1. Measurement of the maximal length of thermal spread. A, Maximal longitudinal distance of coagulation necrosis from the cauterized end of SA samples was measured (dashed arrow, see text for details). The extent of damage to the adventitia and tunica media (bracket) corresponded with the extent of endothelial damage of the tunica intima (short arrow). (Hematoxylin and eosin stain, original magnification $200 \times$.) B, Representative blood vessel with thermal damage affecting predominantly the tunica adventitia (bracket). C, Representative blood vessel with thermal damage affecting the 3 vascular layers: tunica adventitia, media, and intima (bracket). (Hematoxylin and eosin stain, original magnification 100×.)

no injury or thermal spread in 0.25 -mm increments and counted. Thereafter, statistical differences were analyzed with a Wilcoxon rank sum test in SAS 9.2 software (SAS Institute, Inc, Cary, NC). Burst pressure was analyzed with a 2-tailed $t$ test with equal variance within Microsoft Office Excel 2007 software (Microsoft, Redmond, Wash).

\section{RESULTS}

Figure 3 presents the number of arterial conduits experiencing a specific range of thermal spread with the VirtuoSaph (Figure 3, A) and VASOVIEW 6 (Figure 3, B) devices, respectively. From the arterial conduits, 30 of 61 samples had no injury when the VirtuoSaph was used compared with only 3 of 53 samples in the VASOVIEW 6 group. The average thermal spread was significantly shorter $(P<.05)$ in the VirtuoSaph group $(0.42 \pm 0.08 \mathrm{~mm})$ compared with the VASOVIEW 6 group $(1.05 \pm 0.04 \mathrm{~mm})$.

Figure 4 presents the number of venous conduits experiencing a specific range of thermal spread in vein conduits with the VirtuoSaph (Figure 4, A) and VASOVIEW 6 (Figure 4, B) devices, respectively. When the VirtuoSaph was used, 21 of 83 vein samples had no endothelial injury compared with only 3 of 79 samples in the VASOVIEW 6 group. The average length of thermal spread was significantly shorter $(P<.05)$ in the VirtuoSaph group $(0.49 \pm$ $0.05 \mathrm{~mm})$ than in the VASOVIEW 6 group $(0.94 \pm 0.19 \mathrm{~mm})$.

Figure 5 presents the average burst pressure obtained in arterial and venous conduits in each harvester group. Higher burst pressures where observed when the VASOVIEW 6 system was used for both SA and SEV conduits compared with the VirtuoSaph. The average was greater in the VASOVIEW 6 group, and there was considerable overlap between the groups, leading to an insignificant statistical difference.

\section{DISCUSSION}

This study was developed to investigate the extent of thermal graft injury, one of the major concerns during the procurement of vascular conduits for CABG. ${ }^{7,9,16}$ It has been proposed that saphenous vein graft disease is comprised of 3 distinct but interrelated pathological processes: thrombosis, intimal hyperplasia, and atherosclerosis. ${ }^{17}$ Early thrombosis is a major cause of 


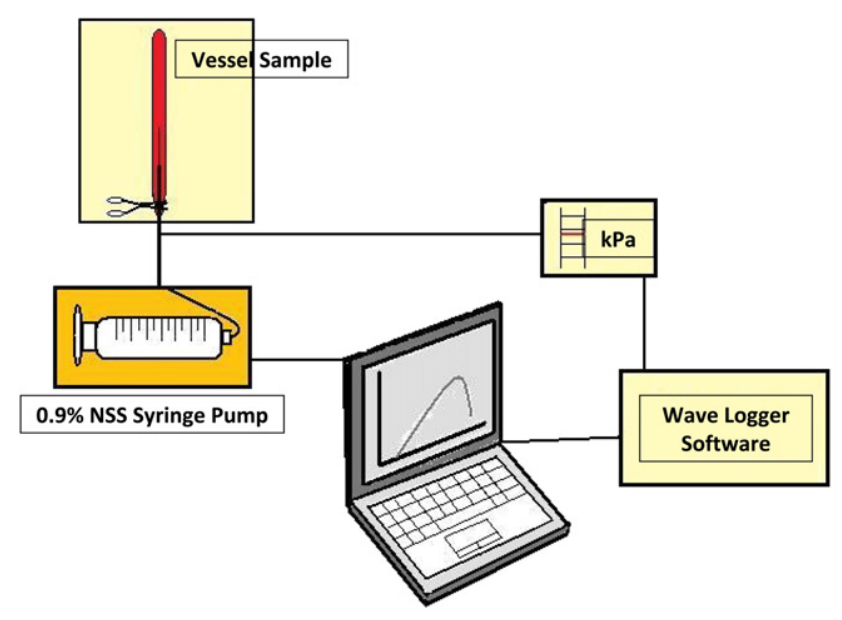

FIGURE 2. Burst pressure test schematic.

vein graft attrition during the first month after bypass surgery, whereas intimal hyperplasia forms a template for subsequent atherogenesis during the remainder of the first year. Thereafter, atherogenesis predominates. Each of these phases is accelerated by the loss of the anatomic and functional integrity of the endothelium during and after grafting. ${ }^{11}$ It is possible that thermal vascular endothelial injury during endoscopic harvesting might increase the risk of early graft failure.

A few similar studies have been published in this area. Allen ${ }^{14}$ measured the total extent of thermal damage (edge to edge) in swine SEVs using the same technique with 3 different bipolar dissectors and reported a longer (2.1-4.4 mm) extent of thermal spread compared with our results $(0.45-1.2 \mathrm{~mm})$. However, he concluded that all performed satisfactorily and that vascular seals need to be consistently reliable to avoid complication-related hemostasis at the donor site. We looked in detail at the burst pressure of the vascular seals for each of the devices we studied and measured objectively this important factor.

Two other published reports evaluated the extent of endothelial damage within the human internal thoracic artery during preparation for $\mathrm{CABG}$ with a harmonic scalpel or high-frequency electrocautery. The study, published by Lamm and associates, ${ }^{18}$ used a scoring system to describe the endothelial damage from scanning electron microscopic images. They concluded that if the distance from the main vessel to the cautery point is greater than $5 \mathrm{~mm}$, the endothelium of the main vessel was confluent and uninjured. If this distance was less than $5 \mathrm{~mm}$, high-frequency electrocautery produced thermal injury on the outer layer of the artery on all grafts with considerable intimal damage. On the other hand, no damage was reported in the harmonic scalpel group. Thus Lamm and associates demonstrated approximately 5 to 10 times greater thermal spread than observed in the current study with either device $(0.42 \pm 0.08 \mathrm{~mm}$ for the VirtuoSaph and $1.05 \pm .04 \mathrm{~mm}$ for the VASOVIEW 6 , Figure 3). This might be due in part to different measurement techniques, but the predominant cause is likely the

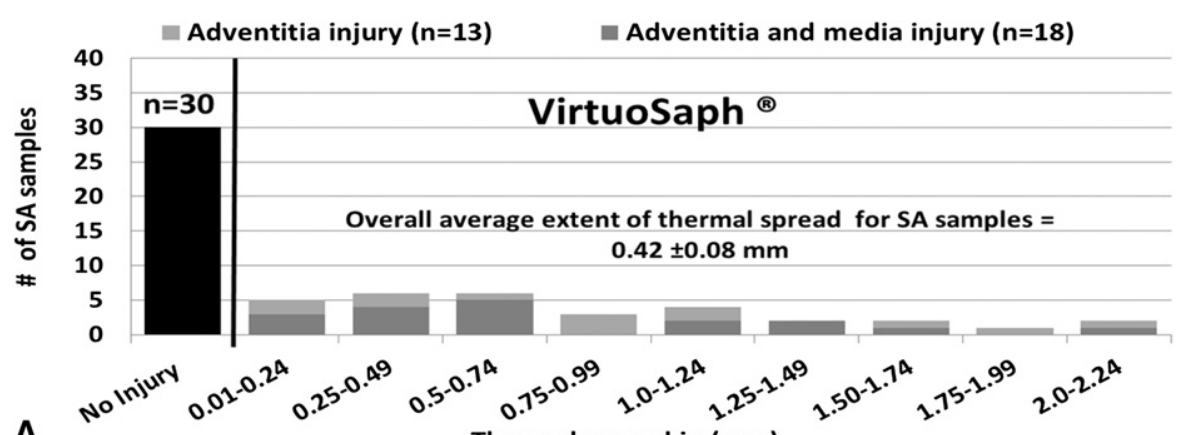

A

Thermal spread in $(\mathrm{mm})$

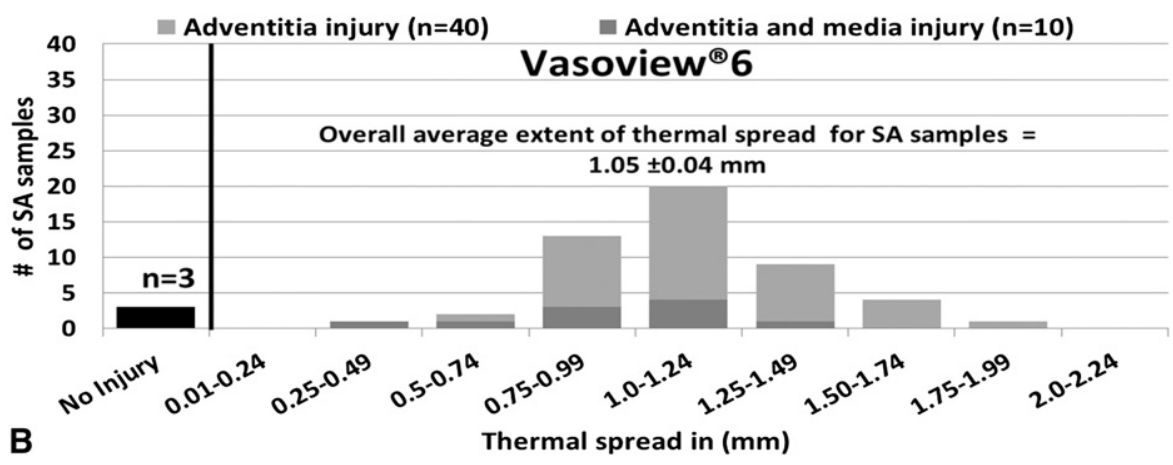

FIGURE 3. Extent of thermal spread in saphenous artery $(S A)$ conduits by using each endoscopic vessel harvesting $(E V H)$ system. The number of arterial conduits experiencing a specific range of thermal spread with the VirtuoSaph (A) and VASOVIEW 6 (B) EVH systems is shown. 

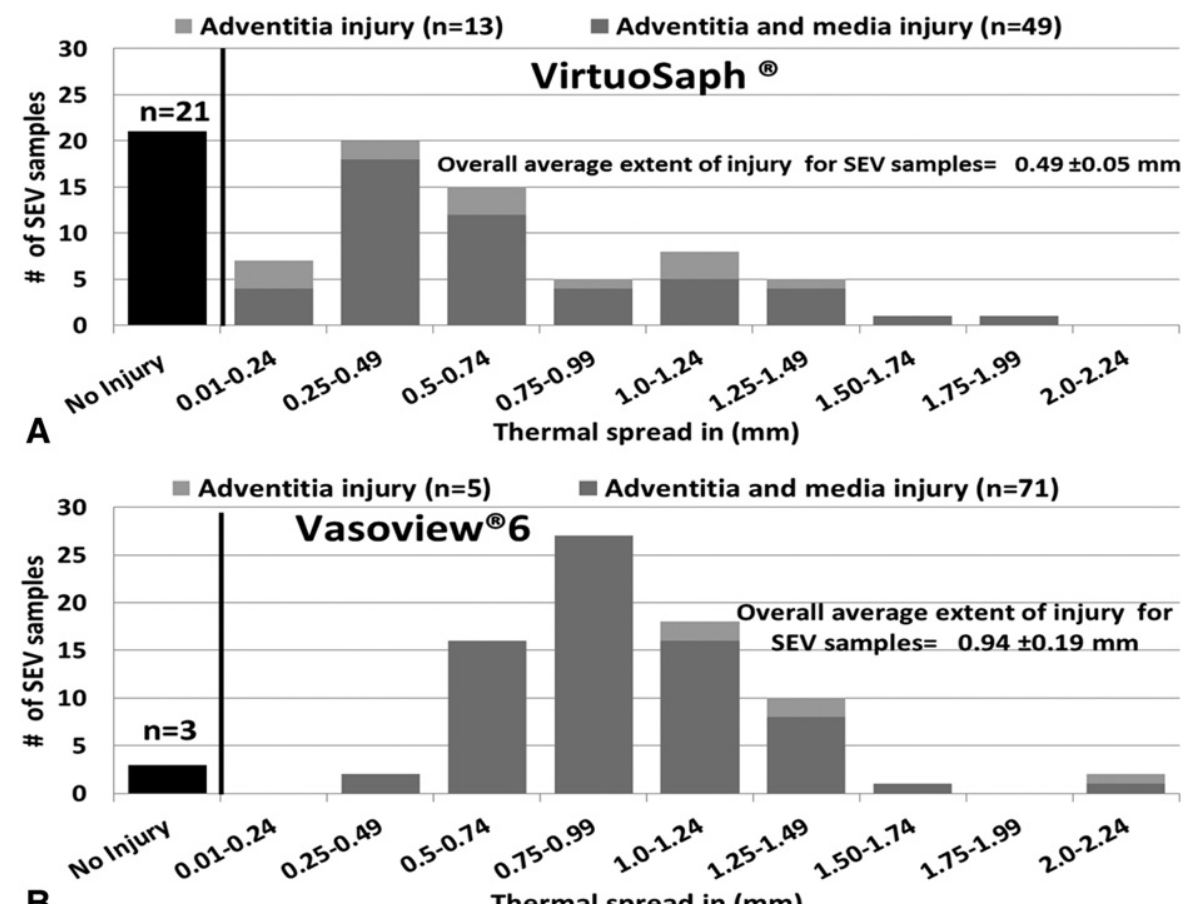

B

Thermal spread in $(\mathrm{mm})$

FIGURE 4. Extent of thermal spread in superficial epigastric vein $(S E V)$ conduits by using each endoscopic vessel harvesting $(E V H)$ system. The number of venous conduits experiencing a specific range of thermal spread with the VirtuoSaph (A) and VASOVIEW 6 (B) EVH systems is shown.

difference in devices used during harvest. Neither of these devices is purposely designed for vessel harvesting.

A second study, published by Higami and coworkers, ${ }^{19}$ evaluated vascular damage during the harvesting of the internal thoracic artery with an ultrasonic scalpel in a pig model. The branches of the artery were cut and assigned to one of 3 groups $(0,1$, and $2 \mathrm{~mm})$ defined by the distance from the main vessel to the cautery point. Tissue sections were evaluated by using computer-image analysis from the cautery point to the boundary between damaged and normal tissue. The lengths of tissue damage were $0.96 \pm$ $0.48 \mathrm{~mm}$ (0-mm group), $0.58 \pm 0.18 \mathrm{~mm}$ (1-mm group),

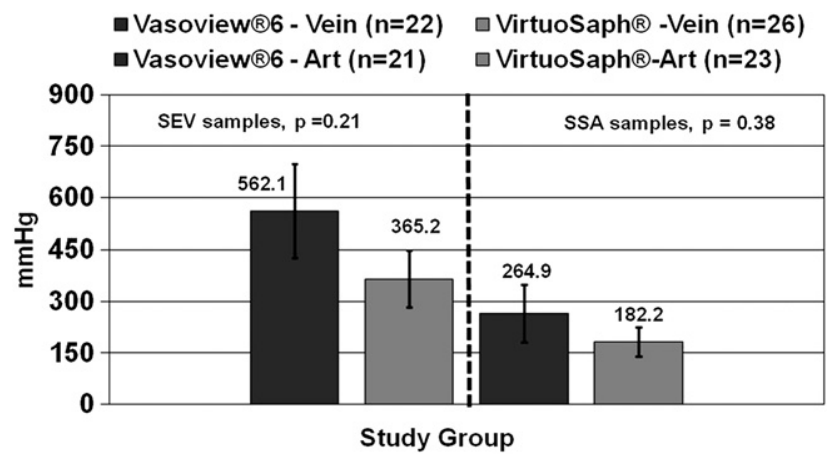

FIGURE 5. Burst pressure in saphenous artery $(S A)$ and superficial epigastric vein $(S E V)$ conduits with each endoscopic vessel harvesting $(E V H)$ system. The average burst pressure obtained in arterial and venous conduits in each harvester group is shown. and $0.63 \mathrm{~mm} \pm 0.27 \mathrm{~mm}$ (2-mm group). All branches from the 0-mm group showed injury of the main vessel, whereas no injury to the main vessel was observed in the other groups. These results demonstrate a similar amount of thermal damage as seen in this study. In addition, $91.7 \%$ of arterial samples in the study by Higami and coworkers had a burst pressure of greater than $350 \mathrm{~mm} \mathrm{Hg}$, a much greater burst pressure than in the current study $(182 \pm 43 \mathrm{~mm} \mathrm{Hg}$ for the VirtuoSaph and $265 \pm 85 \mathrm{~mm}$ $\mathrm{Hg}$ for the VASOVIEW 6, Figure 5). Thus they concluded that this technique is safe when branches are sectioned at least $1 \mathrm{~mm}$ distal to their origin at a sufficiently slow speed.

One additional study, published by Griffith and colleagues, ${ }^{16}$ compared the endoscopic technique and open vein harvest in human subjects. In this study a numeric grading system was developed to estimate the percentage of disruption of the endothelial layer, elastic lamina, medial smooth muscle and connective tissue, and adventitial connective tissue. The authors concluded that no significant differences were observed between techniques but that a mild histologic disruption occurred in all layers of the venous walls, regardless of the method used for harvesting. Unfortunately, this study focused on the difference between 2 harvesting techniques and did not evaluate the length of thermal damage. Thus it did not provide guidelines for endoscopic harvesting to avoid damage to the main vessel.

Overall, results from the current study agree with those of Higami and coworkers. ${ }^{19}$ We found that the length of 
thermal injury was significantly lower for arterial and venous conduits when the VirtuoSaph device was used $(0.42$ \pm 0.08 and $0.49 \pm 0.05 \mathrm{~mm}$, respectively; Figures 3 and 4) compared with that seen in the VASOVIEW 6 group $(1.05 \pm .04$ and $0.94 \pm 0.19 \mathrm{~mm}$, respectively). Clinically, the distance between the vessel keeper/ retractor and the dissector during branch cautery is between 5 and $10 \mathrm{~mm}$. This distance varies per manufacturer but ultimately guarantees a length that will reduce or eliminate injury to the main vessel. However, thermal-spread injury to the main vessel could occur if an inappropriate harvesting technique is used, including too high of wattage setting, inadequate branch setup, or both. Furthermore, although the main vessel is spared, thermal damage between the main vessel and the cauterized branches could have an effect on long-term graft patency. There might be an effect on coagulation in the short-term or hyperplasia in the long-term, but to our knowledge, these issues have not been studied. Thus the clinical effect of zero versus 0.4 to $1.0 \mathrm{~mm}$ of cautery artifact remains unknown.

As discussed above, the VirtuoSaph system resulted in a significantly smaller amount of thermal spread than the VASOVIEW 6 system. The VirtuoSaph also resulted in a smaller average burst pressure, suggesting slightly reduced tunnel-site hemostasis. However, this was not statistically significant for veins or arteries because there is great variability and overlap in the results for the devices. Thus there might be some correlation between the degree of thermal spread and the quality of the seal, but this is of less importance than the variability from seal to seal with any given device.

This study is limited somewhat because an open technique was used rather than the closed tunnel technique that is used in clinical practice. The closed tunnel was not used because it is difficult to perform in swine, especially during the harvesting of the SA, and our goal was to avoid confounding mechanical damage to the vessel. The effect of mechanical stretch and physical trauma caused by EVH tunnel dissection are unknown and warrant further study. Mechanical trauma might be different with EVH than with an open technique. In addition, the long-term patency of the conduits was not assessed. Future work should assess whether these histologic findings correlate with long-term graft patency after CABG.

In conclusion, the length of thermal spread is short, 0.4 to $1.1 \mathrm{~mm}$, and depends on the device used. Careful use of EVH systems by an experienced operator should eliminate injury to the main vessel during harvesting. Clinical protocols should, however, include a minimal length of the cau- terized branch to ensure that thermal spread does not reach the main vessel. The results of this study suggest that at least $1 \mathrm{~mm}$ is sufficient. However, the differences between EVH systems make the standardization difficult, and therefore the manufacturers are encouraged to perform studies to develop their own protocols and recommendations for clinical application.

\section{References}

1. Waqar-Uddin Z, Purohit M, Blakeman N, Zacharias J. A prospective audit of endoscopic vein harvesting for coronary artery bypass surgery. Ann R Coll Surg Engl. 2009;91:426-9.

2. Kiaii B, Moon BC, Massel D, Langlois Y, et al. A prospective randomized trial of endoscopic versus conventional harvesting of the saphenous vein in coronary artery bypass surgery. J Thorac Cardiovasc Surg. 2002;123:204-12.

3. Cheng D, Allen KB, Cohn W, et al. Endoscopic vascular harvest in coronary artery bypass grafting surgery: a meta-analysis of randomized trials and controlled trials. Innovations Technol Techniques Cardiothorac Vasc Surg. 2005;1:61-74.

4. Allen KB, Griffith GL, Heimansohn DA, et al. Endoscopic versus traditional saphenous vein harvesting: a prospective, randomized trial. Ann Thorac Surg. 1998;66:26-32.

5. Allen KB, Heimansohn DA, Robison RJ, et al. Risk factors for leg wound complications following endoscopic versus traditional saphenous vein harvesting. Heart Surg Forum. 2000;3:325-30.

6. Cadwallader RA, Walsh S, Cooper D, Tang T, Sadat U, Boyle J. Great saphenous vein harvesting: a systematic review and meta-analysis of open versus endoscopic techniques. Vasc Endovascular Surg. 2009;43:561-6.

7. Lopes RD, Hafley GE, Allen KB, et al. Endoscopic versus open vein-graft harvesting in coronary-artery bypass surgery. N Engl J Med. 2009;361: 235-44.

8. Yun KL, Wu Y, Aharonian V, et al. Randomized trial of endoscopic versus open vein harvest for coronary artery bypass grafting: six-month patency rates. J Thorac Cardiovasc Surg. 2005;129:496-503.

9. Aziz O, Athanasiou T, Darzi A. Minimally invasive conduit harvesting: a systematic review. Eur J Cardiothorac Surg. 2006;29:324-33.

10. Verrier ED, Boyle EM Jr. Endothelial cell injury in cardiovascular surgery. Ann Thorac Surg. 1996;62:915-22.

11. Motwani JG, Topol EJ. Aortocoronary saphenous vein graft disease: pathogenesis, predisposition, and prevention. Circulation. 1998;97:916-31.

12. Yoshida H, Wu MH, Kouchi Y, Onuki Y, Shi Q, Sauvage LR. Comparison of the effect of monopolar and bipolar cauterization on skeletonized, dissected internal thoracic arteries. J Thorac Cardiovasc Surg. 1995;110:504-10.

13. Brown EN, Kon ZN, Tran R, et al. Strategies to reduce intraluminal clot formation in endoscopically harvested saphenous veins. J Thorac Cardiovasc Surg. 2007;134:1259-65.

14. Allen KB. A comparison of endoscopic thermal dissectors regarding vascular and thermal injury. Surgical Physician Assistant. 2003;9:9-13.

15. Pietrow PK, Weizer AZ, L'Esperance JO, et al. PlasmaKinetic bipolar vessel sealing: burst pressures and thermal spread in an animal model. $J$ Endourol. 2005;19:107-10.

16. Griffith GL, Allen KB, Waller BF, et al. Endoscopic and traditional saphenous vein harvest: a histologic comparison. Ann Thorac Surg. 2000;69:520-3.

17. Alexander JH, Hafley G, Harrington RA, et al. Efficacy and safety of edifoligide, an E2F transcription factor decoy, for prevention of vein graft failure following coronary artery bypass graft surgery: PREVENT IV: a randomized controlled trial. JAMA. 2005;294:2446-54.

18. Lamm P, Juchem G, Weyrich P, Schutz A, Reichart B. The harmonic scalpel: optimizing the quality of mammary artery bypass grafts. Ann Thorac Surg. 2000;69: 1833-5

19. Higami T, Maruo A, Yamashita T, Shida T, Ogawa K. Histologic and physiologic evaluation of skeletonized internal thoracic artery harvesting with an ultrasonic scalpel. J Thorac Cardiovasc Surg. 2000;120:1142-7. 